\title{
Tunneling Characteristics of Au-Alkanedithiol-Au Junctions formed via Nanotransfer Printing (nTP)
}

\author{
Jeremy R. Niskala, ${ }^{\dagger}$ William C. Rice, ${ }^{\ddagger}$ Robert C. Bruce, ${ }^{\dagger}$ Timothy J. Merkel, ${ }^{\dagger}$ Frank Tsui, ${ }^{\ddagger}$ and Wei You, ${ }^{* \dagger}$ \\ ${ }^{\dagger}$ Department of Chemistry, University of North Carolina at Chapel Hill, Chapel Hill, North Carolina 27599-3290, United States \\ ${ }^{\ddagger}$ Department of Physics and Astronomy, University of North Carolina at Chapel Hill, Chapel Hill, North Carolina 27599-3255, \\ United States
}

Supporting Information

\begin{abstract}
Construction of permanent metal-moleculemetal (MMM) junctions, though technically challenging, is desirable for both fundamental investigations and applications of molecule-based electronics. In this study, we employed the nanotransfer printing (nTP) technique using perfluoropolyether (PFPE) stamps to print Au thin films onto selfassembled monolayers (SAMs) of alkanedithiol formed on $\mathrm{Au}$ thin films. We show that the resulting MMM junctions form
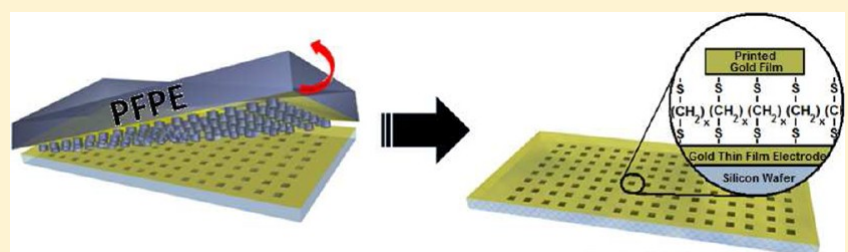

$X=8,10,12$, or 14 permanent and symmetrical tunnel junctions, without the need for an additional protection layer between the top metal electrode and the molecular layer. This type of junction makes it possible for direct investigations into the electrical properties of the molecules and the metal-molecule interfaces. Dependence of transport properties on the length of the alkane molecules and the area of the printed $\mathrm{Au}$ electrodes has been examined systematically. From the analysis of the current-voltage (I-V) curves using the Simmons model, the height of tunneling barrier associated with the molecule (alkane) has been determined to be $3.5 \pm 0.2$ $\mathrm{eV}$, while the analysis yielded an upper bound of $2.4 \mathrm{eV}$ for the counterpart at the interface (thiol). The former is consistent with the theoretical value of $\sim 3.5-5.0 \mathrm{eV}$. The measured $\mathrm{I}-\mathrm{V}$ curves show scaling with respect to the printed Au electrode area with lateral dimensions ranging from $80 \mathrm{~nm}$ to $7 \mu \mathrm{m}$. These results demonstrate that PFPE-assisted $\mathrm{nTP}$ is a promising technique for producing potentially scalable and permanent MMM junctions. They also demonstrate that MMM structures (produced by the unique PFPE-assisted nTP) constitute a reliable test bed for exploring molecule-based electronics.
\end{abstract}

\section{INTRODUCTION}

Abundant research has been dedicated to the study of the electronic properties of single and small collections of molecules, ${ }^{1-4}$ since molecular electronics is not only of fundamental scientific interest but also expected to be rather inexpensive for a variety of applications. In many molecular electronic systems, electrical transport measurements are performed between two metallic electrodes that sandwich the molecules, for example, in a bottom-up approach by making a metal contact to a self-assembled monolayer (SAM) formed on a metal thin film. ${ }^{5-9}$ However, establishing an intimate contact to fragile molecules is not a trivial pursuit. Initial attempts at making contacts to SAMs either by direct thermal vapor deposition $^{10}$ or by deposition onto nanopores ${ }^{11-14}$ have resulted in a high percentage of electrical shorts through the monolayer, as the evaporated atoms/molecules either penetrated into or thermally damaged the SAM. Other techniques for making less permanent contacts on monolayers have been more successful, such as molecular break junctions, ${ }^{15}$ mercury $^{16,17}$ and mercury-drop electrodes, ${ }^{3,18}$ fluid metal eutectic gallium-indium drop junctions, ${ }^{8}$ crossed-wire junctions, ${ }^{19}$ scanning tunneling spectroscopy, ${ }^{9,20}$ and conductive atomic force microscopy (cAFM)..$^{5,21-23}$ Much has been learned from these studies, particularly on electrical transport through molecular layers and metal-molecule interfaces.
However, the results are widely varied, ${ }^{2,24}$ owing primarily to certain ambiguities at the metal-molecule interface, such as uncertainties in the number of molecules contacted, the contact area, and the nature of the contacts.

Permanent metal-molecule-metal (MMM) junctions are quite desirable, but their use has been less common, largely due to the aforementioned challenges. One effective technique to date to prevent shorts is the use of a protective layer over the molecular monolayer, such as organic poly $(3,4$ ethylenedioxythiophene):poly(styrenesulfonate) (PEDOT:PSS $)^{7,25-27}$ or graphene, ${ }^{28}$ prior to thermal deposition of the top metal electrode. However, an additional protection layer and its interfaces with the metal and the molecular monolayer complicate the transport process, thus making it more difficult to interpret the observed current-voltage (I-V) behaviors. $^{27,28}$ A preferred alternative would be a simpler MMM junction with the top metal electrode in direct contact with the molecular monolayer. One promising method that produces this architecture is nanotransfer printing (nTP). ${ }^{29,30}$

Nanotransfer printing provides an inexpensive, straightforward method to transfer potentially scalable arrays of thin metal films onto SAMs without damaging the monolayer beneath. In

Received: March 16, 2012

Published: June 21, 2012 
principle, metal thin films deposited onto elastomeric polymer stamps can be transferred to any surface as long as the metal thin film has a higher affinity for the receiving substrate than that of the stamp. In many cases, the receiving substrate utilizes a functionalized monolayer to chemically bind to the printed film to assist the transfer process. Improved transfer techniques of materials onto various SAM-coated substrates, such as silicon, $^{30-33}$ glass and plastic, ${ }^{30,34}$ and $\mathrm{n}^{+}$doped GaAs, ${ }^{6,35-37}$ have been reported. However, there are only a few reports of nTP onto SAMs on metal surfaces. ${ }^{38-40}$

Recently, we successfully used cross-linked perfluoropolyether (PFPE) elastomeric stamps in the transfer printing of $\mathrm{Au}$ thin films onto SAM-coated metal thin films. ${ }^{40}$ Reproducible tunneling I-V characteristics were obtained from $200 \mathrm{~nm}$ $\mathrm{Au}$-decanedithiol-Au features, indicating the viability of using this nondestructive approach to investigate the electrical properties of molecular assemblies. In this current article, we report a systematic study of the surface and electrical properties of transfer printed $\mathrm{Au}$-alkanedithiol-Au MMM junctions probed by cAFM. Current-voltage (I-V) measurements were carried out and analyzed as a function of (a) the molecular length from octanedithiol (C8) to tetradecanedithiol (C14) and (b) the nTP contact size from $80 \mathrm{~nm}$ to $7 \mu \mathrm{m}$ in lateral dimensions. Several hundred individual junctions from a large number of separately fabricated substrates were examined in order to distinguish extrinsic effects and establish statistically relevant intrinsic results. Our aim is to examine the viability of the nTP technique for producing stable and reproducible MMM junctions and to elucidate the nature of the molecular junctions including the molecular barriers and their interfacial states with metal contacts. The current-voltage characteristics of the printed MMM junctions exhibit nonresonant tunneling behavior. Simmons model analysis of the measurements reveals a tunneling barrier height of $3.5 \pm 0.2 \mathrm{eV}$ for the alkane, which is among the highest experimental values reported for linear alkanes and consistent with the theoretical values of $3.5-5.0 \mathrm{eV}$ for these molecules. ${ }^{41}$

\section{EXPERIMENTAL SECTION}

The nTP process consists of several integral steps, including SAM formation on the bottom metal contact, preparation of PFPE stamps, and transfer printing, as shown in Figure 1. These are described as follows.

Bottom Contact and SAM Formation. Thermal evaporation of metal films on $\mathrm{Si}$ wafers was performed using an evaporator contained within a nitrogen-filled glovebox so that the freshly evaporated thin films can be removed from the evaporator chamber under inert conditions for SAM formation. Prior to Au deposition, each Si wafer (with $1 \mu \mathrm{m}$ thick surface oxide) was first cleaned in a solution of DI water, ammonium hydroxide $(14.8 \mathrm{M})$, and hydrogen peroxide $(30 \%$, $\mathrm{H}_{2} \mathrm{O}$ ) in a 2:1:1 ratio for $15 \mathrm{~min}$. It was then rinsed thoroughly with water and ethanol and dried with a stream of nitrogen gas, followed by UV/ozone cleaning for $20 \mathrm{~min}$. After cleaning, a $3 \mathrm{~nm}$ Ti adhesion layer was first deposited at $1 \AA / \mathrm{s}$ followed by Au deposition $(60 \mathrm{~nm})$, which started at a rate of $1 \AA / \mathrm{s}$ for the first $1-3 \mathrm{~nm}$ and then quickly increased to $10 \AA / \mathrm{s}$ for the duration of the deposition. The higher deposition rates $(>5 \AA / \mathrm{s})$ for Au have been shown to result in smoother films, ${ }^{40}$ which are critical for high quality and reproducible nTP.

Monolayer formation on $\mathrm{Au}$ was performed using the welldocumented and thoroughly characterized $\mathrm{Au}$-thiol attachment chemistry. ${ }^{42-45}$ Specifically, SAMs were formed from alkanedithiols following the literature procedures that have been shown to form vertically aligned, densely packed monolayers on $\mathrm{Au}^{45-48}$ Following $\mathrm{Au}$ deposition onto the silicon wafer, the Au thin film substrate was
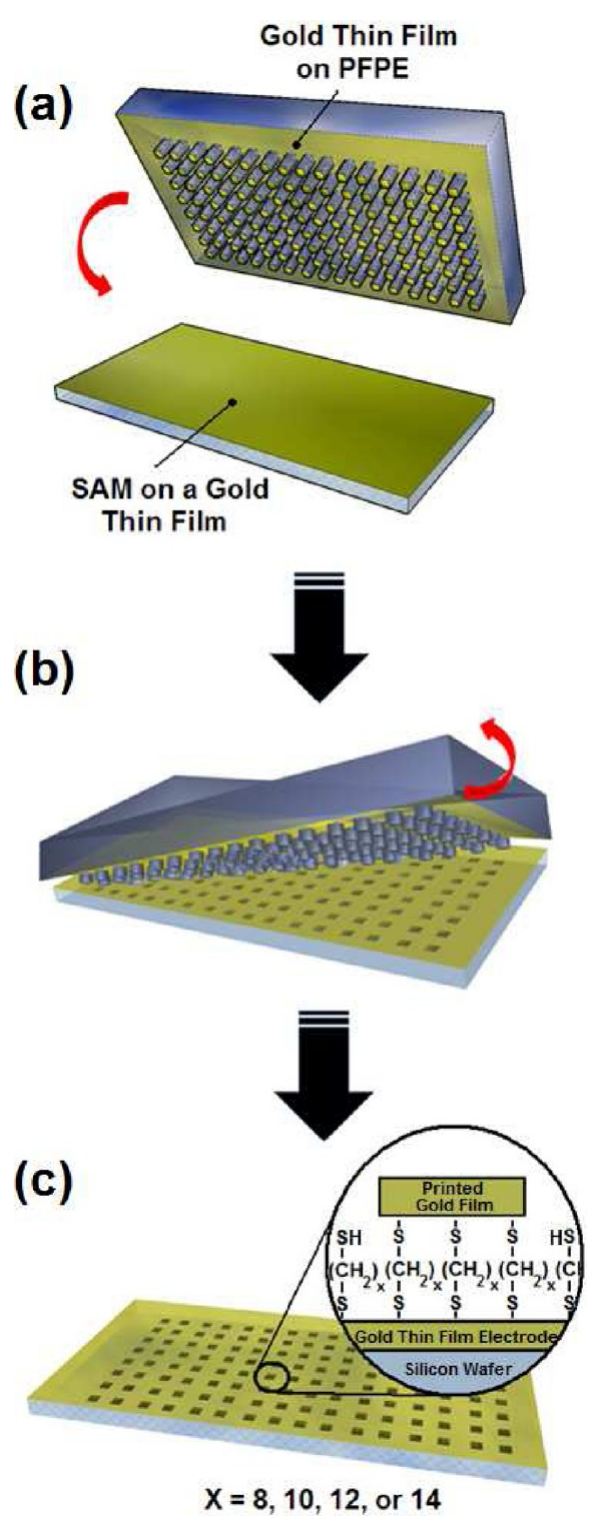

Figure 1. Schematic diagram of the nTP process. (a) A Au-coated (20 $\mathrm{nm}$ ) PFPE stamp with predefined raised features is brought into contact with a SAM-coated Au electrode. After allowing the PFPE/Au to react with the exposed thiol, (b) the stamp is slowly peeled away from the Au substrate thus leaving behind (c) a well-defined array of $\mathrm{Au}-$ molecule- $\mathrm{Au}$ junctions.

immediately immersed in a solution of alkanedithiol in ethanol (all within the glovebox and stored in the dark). Octanedithiol and decanedithiol were purchased from Alfa Aesar and were used as received, and dodecanedithiol and tetradecanedithiol were synthesized using previously reported synthetic procedures. ${ }^{7}$ Octanedithiol and decanedithiol samples were prepared from $30 \mathrm{mM}$ solutions, and dodecanedithiol and tetradecanedithiol were from $15 \mathrm{mM}$ solutions. Dodecanedithiol and tetradecanedithiol solutions were heated to 70 ${ }^{\circ} \mathrm{C}$ prior to monolayer formation to fully dissolving the dithiol molecule. After $24 \mathrm{~h}$, each sample was removed from the alkanedithiol solution, sonicated in THF for $30 \mathrm{~s}$ to remove any physisorbed molecules, rinsed with ethanol, dried under a $\mathrm{N}_{2}$ stream, and further dried under vacuum ( $\sim 1 \mathrm{mbar})$ for $30 \mathrm{~min}$.

PFPE Stamp Preparation and Characterization. PFPE stamps with an array of raised features were replicated from PFPE molds with a analogous array of wells that was produced using standard photolithography techniques. ${ }^{40,49}$ Liquid PFPE prepolymer (Liquidia Technologies) was drop-cast onto a planar mold and cured by UV 
illumination (at $125 \mathrm{~mW} / \mathrm{cm}^{2}$ ) for $4 \mathrm{~min}$. After curing, the PFPE stamp with a raised array of circular or square features was peeled away from the mold. Each newly formed PFPE stamp was then cut to $\sim 1$ $\mathrm{cm} \times 1 \mathrm{~cm}$ squares each of which was then taped to a glass slide using double-sided tape with the arrays exposed, directed away from the glass slide. Taping to the slide was done for ease of handling during vapor deposition. Deposition was performed using the same equipment detailed above. Twenty nanometers of $\mathrm{Au}$ was thermally deposited starting at $1 \AA / \mathrm{s}$ for the first $1 \mathrm{~nm}$ of thickness and then the rate was increased to $10 \AA / s$ for the final duration of the deposition. An elevated $\mathrm{Au}$ deposition rate is needed to ensure metal accumulation on the PPFE surface, owing to its low surface energy leading to low adsorption and high migration rates for metals.

Two types of PFPE were used, a low-viscosity PFPE and highviscosity PFPE. Elastic moduli of the PFPE were obtained from the stress-strain measurements via an Instron 5566 analyzer operated under ambient conditions.

Nanotransfer Printing. After the metal deposition, the PFPE stamps were removed from the glass slides. This step is necessary to take full advantage of the stamp's flexibility during the printing process. As shown in Figure 1a, the stamp was placed on top of the SAMcoated electrode with the array of features brought into contact with the SAM. In addition to the stamp's weight, a slight tapping on the back of the PFPE stamp was determined to be necessary for establishing optimum contact between the Au film and the SAM/ electrode. The whole stack (bottom Au electrode/SAM/Au PFPE stamp) was placed in a vacuum chamber $\left(\sim 10^{-6}\right.$ mbar $)$ for $1 \mathrm{~h}$ to remove any air trapped between the SAM and the $\mathrm{Au}$ film to be transferred. After removing the stack from the vacuum, the stamp was peeled away from the substrate, leaving behind a patterned array of $\mathrm{Au}$ pads on top of the SAM/electrode, as shown in Figure $1 b$ and c.

Monolayer Characterization. The SAMs were characterized by X-ray photoelectron spectroscopy (XPS) and electrochemical measurements following previously reported literature procedures. ${ }^{45}$ XPS characterization was performed using a monochromatic $\mathrm{Al} \mathrm{K} \alpha$ source (source and detector from Kratos) with a power output of 150 W. For all substrates, signal intensity was optimized by adjusting substrate height relative to the source such that the Au $4 \mathrm{f}^{7 / 2}$ signal was maximized. Sulfur $2 p$ spectra were obtained by averaging 6 sweeps at $800 \mathrm{~ms}$ per point with $0.1 \mathrm{eV}$ resolution. The spectra were deconvoluted by first subtracting a Shirley background and then assigning a combination of Lorenzian and Gaussian (Voigt) functions. Spin-orbit separation between $S 2 p^{3 / 2}$ and $S 2 p^{1 / 2}$ signals was set to $1.18 \mathrm{eV}$, and two $\mathrm{S} 2 \mathrm{p}$ signals were assigned: one attributed to bound thiol groups $(\sim 162 \mathrm{eV})$ and one attributed to a combination of unbound thiols and disulfides $(\sim 163.5 \mathrm{eV})$.

A standard three-electrode electrochemical cell setup was used to conduct reductive desorption measurements of the monolayers. An $\mathrm{Ag} / \mathrm{AgCl}$ electrode $(3 \mathrm{M} \mathrm{KCl})$ and platinum wire were used as reference and counter electrode, respectively. All potentials referenced are referred to this $\mathrm{Ag} / \mathrm{AgCl}$ electrode. A solution of $0.1 \mathrm{M} \mathrm{KOH}$ and $1.0 \mathrm{M} \mathrm{KNO}_{3}$ was prepared in pure water (resistance $>18 \Omega$ ) and degassed with argon for several minutes prior to the measurement. Monolayer thiols were reductively desorbed from the $\mathrm{Au}$ surface by applying a bias from 0 to $-2 \mathrm{~V}$ at a rate of $50 \mathrm{mV} / \mathrm{s}$ under a blanket of argon at room temperature. To obtain the overall charge density (the "q-value") for the reductive desorption, the measured current density was integrated between -0.9 and $-1.2 \mathrm{~V}$ (the voltage region where reductive desorption was observed). A straight line connecting the data points at -0.9 and $-1.2 \mathrm{~V}$ was used to approximate a region under the curve, where electrons were generated by the thiol removal.

Using XPS and electrochemical reductive desorption techniques, the SAMs were determined to be dense and vertically aligned with densities comparable to those reported in previous work, ${ }^{45}$ as shown Figures S1 and S2 in the Supporting Information.

Printed Feature Characterization. The surfaces of the printed features were characterized by scanning electron microscopy (SEM) using a FEI Helios 600 Nanolab and by AFM using an Asylum MFP$3 \mathrm{D}$. AFM topography measurements were performed in AC mode under ambient conditions $\left(T=21{ }^{\circ} \mathrm{C}, \mathrm{RH}=45 \%\right)$, using Si cantilevers
(BudgetSensors, Tap300Al) with resonance frequencies of approximately $300 \mathrm{kHz}$, a force constant of $\sim 40 \mathrm{~N} / \mathrm{m}$, and tip radii of less than $10 \mathrm{~nm}$. Root-mean-square surface roughness $\left(R_{\mathrm{rms}}\right)$ values were obtained from the topography measurements. To obtain sufficient statistics for the surface roughness analysis, multiple AFM topography scans were taken on several different printed pads from at least 3 separately fabricated substrates, with each scan typically over $2 \mu \mathrm{m} \times 2$ $\mu \mathrm{m}$ area at a scan rate of $0.5 \mathrm{~Hz}$. Specifically, for pads $>2 \mu \mathrm{m}$ in dimensions, the $2 \mu \mathrm{m} \times 2 \mu \mathrm{m}$ scan was taken within the entire printed feature, and the $R_{\mathrm{rms}}$ value was determined using the entire scan, whereas for smaller pads, the $R_{\mathrm{rms}}$ values were determined from selected areas in the middle of the printed features away from the edges.

Conductive AFM experiments were carried out in either conductive imaging at a constant sample bias of $+1.0 \mathrm{~V}$ or $\mathrm{I}-\mathrm{V}$ mode using the same AFM instrument in contact mode with a constant force of $\sim 10$ $\mathrm{nN}$. Custom made Au-coated Si cantilevers with tip radii larger than 25 $\mathrm{nm}$ were used for the study, in order to minimize contact resistance by maximizing the contact area. These were fabricated by sputter deposition of $25 \mathrm{~nm} \mathrm{Ti}$ and $150 \mathrm{~nm}$ Au onto standard Tap300Al Si cantilevers. Their spring constants were determined to be $\sim 73 \mathrm{~N} / \mathrm{m}$. The AFM measurements were reduced and analyzed using OriginLab, Minitab (version 16.0) and Igor Pro (version 6.05).

Analysis of I-V Curves: Simmons Model. The measured I-V curves were analyzed using the Simmons model. ${ }^{50,51}$ In this model, the tunneling current through a thin layer of insulator between two metal contacts is given by

$$
\begin{aligned}
I= & \left(\frac{q A}{4 \pi^{2} \hbar d_{0}^{2}}\right)\left\{\left(\Phi-\frac{q V}{2}\right) \exp \left[-\frac{2(2 m)^{1 / 2}}{\hbar} \alpha\left(\Phi-\frac{q V}{2}\right)^{1 / 2} d_{0}\right]\right. \\
& \left.-\left(\Phi+\frac{q V}{2}\right) \exp \left[-\frac{2(2 m)^{1 / 2}}{\hbar} \alpha\left(\Phi+\frac{q V}{2}\right)^{1 / 2} d_{0}\right]\right\}
\end{aligned}
$$

where $A$ is the top printed contact/junction area, $m$ and $q$ are the respective electron mass and charge, $d_{0}$ is the distance between the two metal contacts, $\Phi$ is the barrier height of the insulator, $\alpha$ is an adjustable parameter to account for the effects of barrier shape and the electron effective mass within the insulator, and $V$ is the bias. At low bias, the Simmons equation can be simplified, giving the zero-bias resistance as

$$
R_{0}=\left(\frac{8 \pi^{2} \hbar d_{0}}{A q^{2} \beta_{0}}\right) \exp \left(\beta_{0} d_{0}\right)
$$

where the tunneling decay coefficient $\beta_{0}$ is given by

$$
\beta_{0}=\frac{2 \sqrt{2 m}}{\hbar} \alpha \sqrt{\Phi}
$$

To further examine the molecular tunneling barrier, the $\mathrm{I}-\mathrm{V}$ measurements were fit to eq 1 using a Levenberg-Marquardt $\chi^{2}$ minimization program (in Igor Pro). Specifically, $\Phi, \alpha$, and $d_{0}$ were the adjustable fitting parameters for the effective barrier height, shape, and width, respectively, whereas the contact area $A$ was kept constant and was defined by the area of the top printed contact. Even with $A$ fixed, the minimization algorithm not only converges slowly but also tends to reach various local $\chi^{2}$ minima, owing in part to the presence of correlations between the parameters. To overcome the latter limitation, a statistical approach was employed, such that for each individual $\mathrm{I}-\mathrm{V}$ curve, multiple fits were performed systematically with the initial values for each of the fitting parameters scanned over the respective range of literature values. The resulting parameters from the best fits (i.e., fits with the lowest $\chi^{2}$ values), and their distributions (Figure S3 in Supporting Information) were obtained, from which the best fit values for $\Phi, \alpha$, and $d_{0}$ were determined. 

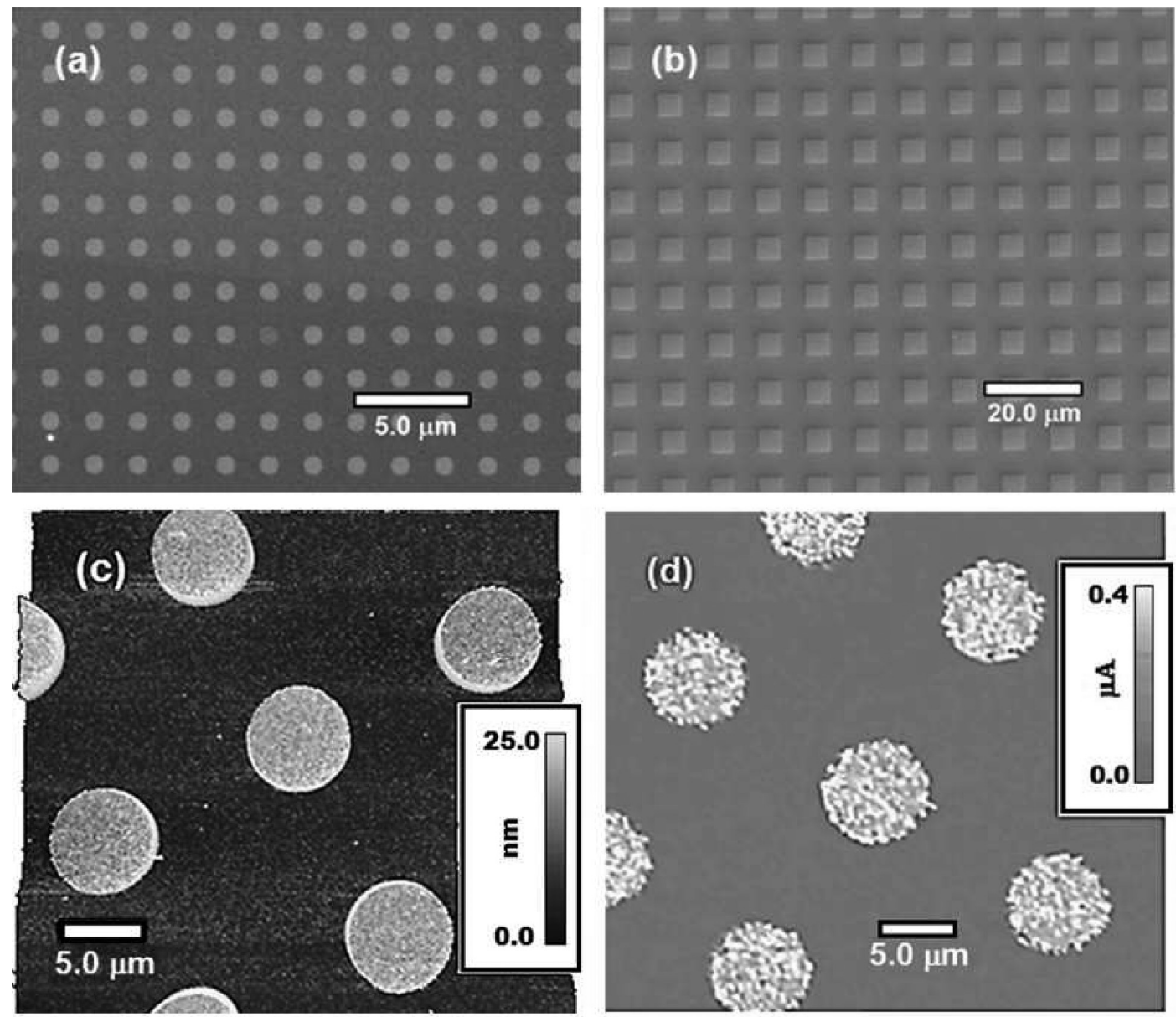

Figure 2. Typical images of nTP arrays of Au-decanedithiol-Au junctions. SEM images of (a) $1 \mu \mathrm{m}$ diameter junctions and (b) $5 \mu \mathrm{m} \times 5 \mu \mathrm{m}$ square junctions. AFM images of $7 \mu \mathrm{m}$ diameter junctions: (c) topography and (d) conductive mapping at a sample bias of $+1.0 \mathrm{~V}$.
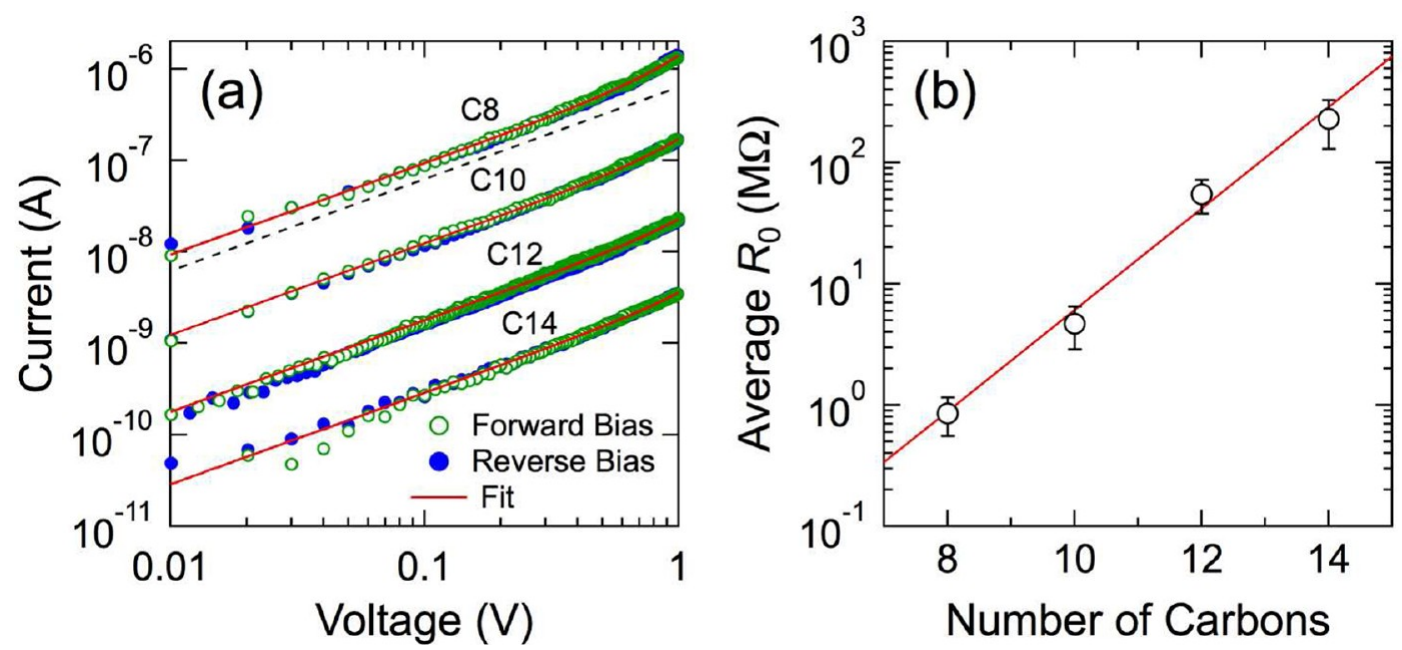

Figure 3. Characteristic $\mathrm{I}-\mathrm{V}$ behavior for $200 \mathrm{~nm}$ diameter $\mathrm{nTP}$ contacts versus molecular length in Au-alkanedithiol SAM-Au junctions. (a) Representative I-V curves (absolute values) for various alkane molecules. Open and closed circles indicate forward and reverse bias, respectively; the solid lines correspond to the Simmons model fits as described in the text. The dashed line corresponds to the linear behavior. (b) Averaged zero-bias resistance versus molecular length of alkane. The solid red line is a linear fit to the semilog plot.

\section{RESULTS AND DISCUSSION}

Using the nTP technique as detailed in the Experimental Section, $20 \mathrm{~nm}$ thick $\mathrm{Au}$ electrodes with a wide range of lateral dimensions were printed from PFPE stamps onto alkanedithiol SAM coated Au thin films in high fidelity and over a large area (several square millimeters), as shown in Figure $2 \mathrm{a}$ and $\mathrm{b}$. The strong chemical affinity between the exposed terminal thiol of the SAM layer and the Au film on the PFPE stamp assisted the transfer process, thus allowing the Au pads to be easily transfer printed onto the SAM. The printed Au pads showed no signs of 
degradation after sonicating in THF for $1 \mathrm{~min}$ and remained bound to the substrate following the scotch tape adhesion test, a widely used and generally accepted empirical technique to ensure sulfur-metal bond formation from transfer printing. ${ }^{6,31,35,37}$ These pieces of evidence suggest bond formation between the printed top Au film and the SAM during the nTP process.

AFM topography and conductive mapping measurements show that the nTP Au surfaces are smooth and the electrical properties of the printed MMM junctions are stable, as shown in Figure $2 \mathrm{c}$ and $\mathrm{d}$. I-V curves measured from the same junctions immediately after sample fabrication and after exposure to the atmosphere for several days did not change, indicating that the molecular junctions are stable over time. Up to $96 \%$ of the MMM junctions tested per substrate exhibited "tunneling-like" I-V characteristics. Most of the remaining 4\% of junctions were electrically shorted, while occasionally, "outliers" with resistance more than 1000 times higher than the average resistance were observed. Shorted junctions evidently occur as a result of the top electrodes being pressed into the bottom electrode during printing. The high resistance outliers, on the other hand, are likely due to the presence of impurities, defects, and/or poor contacts at the thiol/Au interfaces, leading to additional interfaces/tunneling barriers and thus the significantly reduced electron transmittance. Since both the shorted and the highly resistive junctions are defective, they do not exhibit intrinsic molecular conductance, so they were discarded and not used in the Simmons analysis.

Dependence of Tunneling Characteristics on Molecular Length. The I-V behaviors of the $\mathrm{Au}-$ alkanedithiol-Au junctions fabricated by nTP as measured by cAFM exhibit the characteristics of nonresonant tunneling, consistent with previous studies. To study the dependence on molecular length, $200 \mathrm{~nm}$ diameter nTP contacts on SAMs were chosen. As shown in Figure 3a, typical I-V curves are linear at low bias and become exponential at high bias. They show very good symmetry with respect to the direction of the bias voltage (open and closed symbols in Figure 3a), indicating that the two metal-thiol interfaces are nearly identical. For any given bias, the current exhibits exponential dependence on molecule length. The values of zero-bias resistance, $R_{0}$, have been determined from the slopes of the linear $\mathrm{I}-\mathrm{V}$ behavior at low bias and averaged for a given top contact size and molecular length, each from at least 50 independent junctions. The result for $200 \mathrm{~nm}$ diameter contacts is shown in Figure $3 \mathrm{~b}$, which clearly agrees with eq 2 , demonstrating the exponential dependence on molecular length.

The measured I-V curves were analyzed using the Simmons model, as described in the Experimental Section. The results of the analysis for junctions with $200 \mathrm{~nm}$ diameter nTP contacts are shown in Figure 4. The barrier height $\Phi$ increases with increasing molecular length, and as the carbon chain becomes longer than $\mathrm{C} 12$, the barrier height begins to saturate toward a value of $3.5 \mathrm{eV}$. The barrier shape parameter $\alpha$, in contrast, exhibits an opposite trend with lengthening carbon chain: it decreases and then appears to saturate toward a value of 0.57 . This observation suggests that the alkane chain has a higher tunneling barrier with a different shape (lower $\alpha$ value) than the thiol end groups. Our analysis also yields an expected linear dependence of the spacer distance on the length of the carbon chain. The linear behavior for $d_{0}$ not only lends credence to the analysis but also allows quantitative values for the size of thiol $\left(d_{\mathrm{th}}\right)$ and the projected spacing of each $\mathrm{C}-\mathrm{C}$ perpendicular to

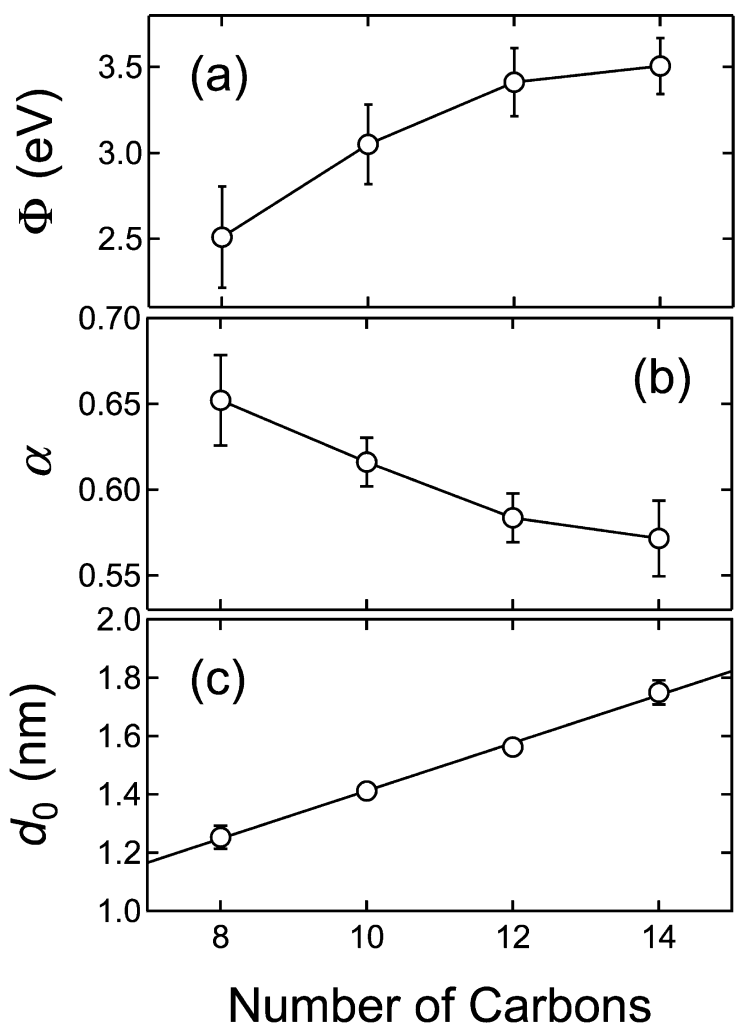

Figure 4. Results of Simmons analysis: dependence of tunneling parameters on the length of alkane backbone for Au-alkanedithiolAu junctions with $200 \mathrm{~nm}$ diameter nTP Au contacts. The line in (c) is a linear fit to the points.

the Au electrodes $\left(d_{\perp}\right)$ to be determined, i.e., $d_{0}=N d_{\perp}+2 d_{\mathrm{th}}$ where $N$ is the number of carbons. Specifically, the vertical intercept of the linear fit (line in Figure 4c) at zero number of carbons $(N=0)$ gives a value of $0.60 \pm 0.04 \mathrm{~nm}$ for $2 d_{\mathrm{th}}$ and thus $0.30 \pm 0.02 \mathrm{~nm}$ for $d_{\mathrm{th}}$, while the corresponding slope yields a projected $\mathrm{C}-\mathrm{C}$ spacing perpendicular to the bottom Au electrode $\left(d_{\perp}\right)$ of $0.081 \pm 0.008 \mathrm{~nm}$. The former is in good agreement with literature values, but the latter is roughly $0.02-$ $0.05 \mathrm{~nm}$ shorter than previously documented values. ${ }^{26,52}$ It is generally accepted that alkanethiolates form ordered $(\sqrt{3} \times$ $\sqrt{3)} \mathrm{R} 30^{\circ}$ domains on $\mathrm{Au}$ films with the alkane chains oriented roughly $26-31^{\circ}$ from normal (Figure 5a), as a result of van der Waals interactions. ${ }^{43,53-56}$ Various calculated values for the unit length of alkyl along the alkane chain $d_{\|}$have been reported, ${ }^{26,52}$ ranging from 0.128 to $0.109 \mathrm{~nm}$. Using these values combined with $d_{\perp}$ from our analysis, the corresponding tilt angle $\theta$ for the alkane chain resulting from the nTP process is determined (from $\cos \theta=d_{\perp} / d_{\|}$) to be between $41^{\circ}$ and $50^{\circ}$. The noticeably larger value is perhaps not surprising given the relatively forceful nature of the transfer printing process.

One would expect that the SAM becomes compressed under the printing force when the top Au electrode is pressed onto the receiving monolayer during printing. It is also possible for the SAM layer to collapse during printing, leading to the "gauche-like" defect or other monolayer defects, ${ }^{9}$ but since this would lead to a very large number of shorted devices, this scenario is inconsistent with the small number of shorts measured in our MMM junctions and unlikely for the densely packed, crystalline-like SAMs produced in this study. The most probable SAM deformation in this system would be a systematic increase in the SAM's tilt angle from normal 
(a)
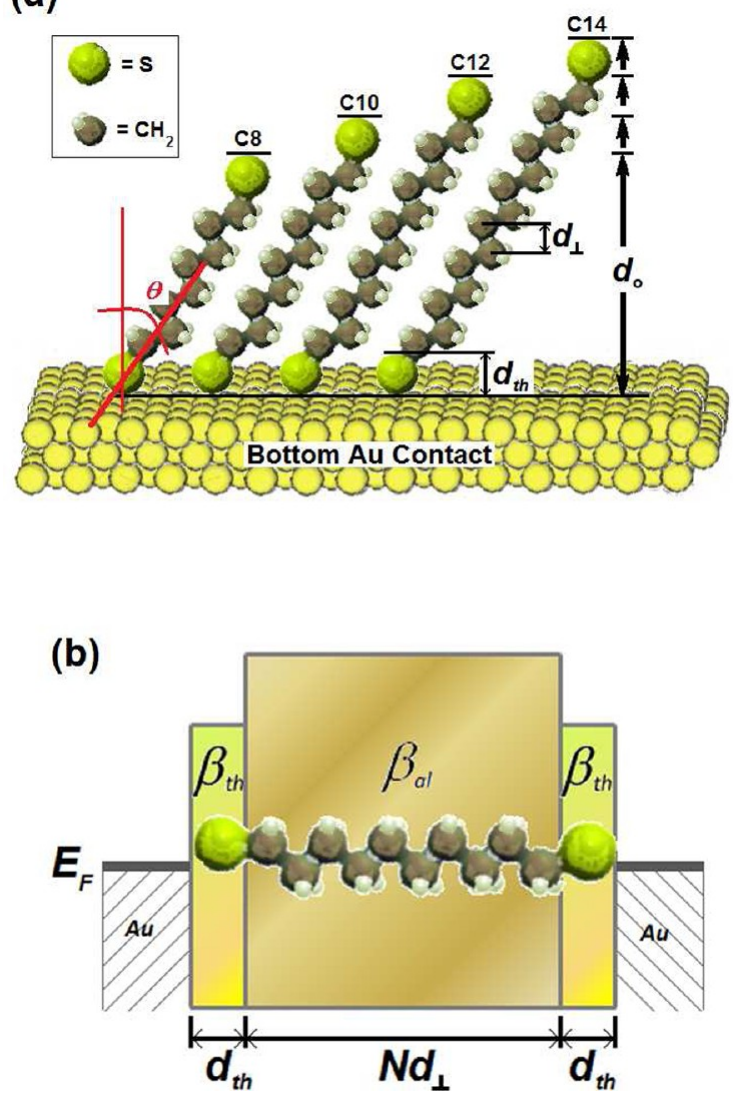

Figure 5. Schematic diagrams of alkanedithiol monolayers on Au. (a) C8-C14 alkandithiols on Au surface tilted by angle $\theta$ with dimensions $d_{\mathrm{th}}, d_{\perp}$, and $d_{0}$ shown and defined in the text. (b) Multibarrier tunneling model for alkanedithiol. ${ }^{27,60} E_{\mathrm{F}}$ is the Fermi energy of the $\mathrm{Au}$ electrodes.

under the applied printing force. Several prior studies have demonstrated presence of an ordered tilt-chain deformation in SAM, where a SAM layer is compressed toward the substrate in a lever-like action under an externally applied force. ${ }^{22,57-59}$

The effects of the thiol and alkane portions of the molecule on the tunneling barrier can be further analyzed and separated using a simple multibarrier model, where the alkane barrier is sandwiched between two identical thiol barriers, as shown in Figure $5 b^{27,60}$ For coherent tunneling through a series of barriers, the overall decay coefficient $\beta_{0}$ is related to the individual decay coefficients, $\beta_{\mathrm{al}}$ and $\beta_{\mathrm{th}}$ for alkyl and thiol, respectively, through the expression

$$
\beta_{0} d_{0}=\beta_{\mathrm{th}} d_{\mathrm{th}}+\beta_{\mathrm{al}} N d_{\perp}+\beta_{\mathrm{th}} d_{\mathrm{th}}
$$

Here, the effect of dielectric constants is neglected. By substituting eq 4 into the expression for $R_{0}$ in eq 2, it is evident that the slope of the linear fit in Figure $3 b$ corresponds to the decay coefficient for alkyl $\beta_{\text {al }}$ with a value of $0.98 \pm 0.11$ per $d_{\perp}$ or $1.21 \pm 0.18 \AA^{-1}$ (using $d_{\perp}$ from our analysis above). This value is consistent with those documented in the literature. ${ }^{2,14,23,24,61}$ The values for the tunneling exponent, $\beta_{0} d_{0}$, have also been determined from the Simmons fits (Figure 4 ), and the resulting dependence of $\beta_{0} d_{0}$ versus the length of carbon chain is shown in Figure 6 for $200 \mathrm{~nm}$ diameter nTP junctions. From the line fit of the behavior, specifically from the slope and the vertical intercept of the fit, the respective values for $\beta_{\text {al }}$ (again using $d_{\perp}$ from our analysis) and $\beta_{\text {th }}$ have been

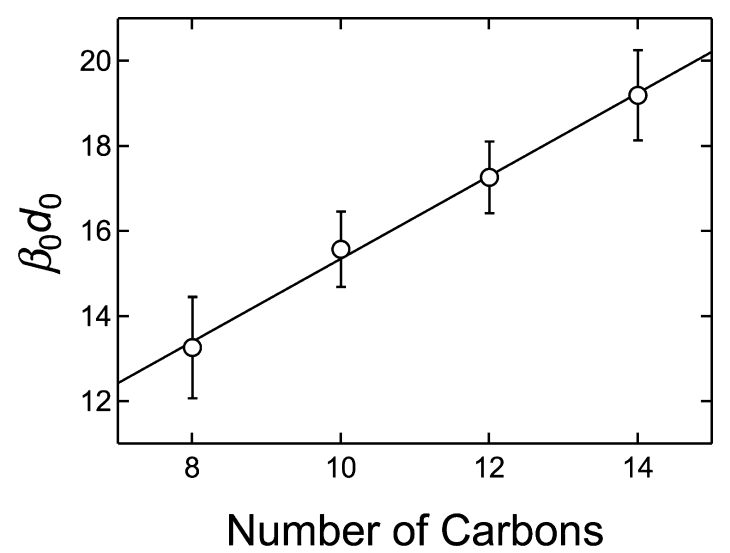

Figure 6. Tunneling exponent versus the length of alkane chain. The line is a linear fit of the points.

determined. The results are $\beta_{\mathrm{al}}=1.16 \pm 0.10 \AA^{-1}$ and $\beta_{\mathrm{th}}=1.00$ $\pm 0.14 \AA^{-1}$. The former decay coefficient agrees with our analysis for $R_{0}$ (above) and with literature values. ${ }^{62,63}$ The excellent agreement further demonstrates the validity of our Simmons fits. However, the value for $\beta_{\text {th }}$ is significantly higher than a previously reported value of $0.05 \AA^{-1.52}$ The effects associated with a very low $\beta_{\text {th }}$ are described in the discussion of tunneling parameters below.

Analysis of the nTP MMM junctions versus the length of alkane molecules has enabled us to deconvolute the tunneling parameters for alkyl and thiol molecules. By extrapolating the behaviors shown in Figure 4a and b, respective values for the barrier height and the shape parameter $\alpha$ for alkyl and thiol have been estimated. For alkyl, we observed a barrier height of $3.5 \pm 0.2 \mathrm{eV}$ and a value for $\alpha$ of $0.6 \pm 0.1$, and correspondingly an upper bound of $2.4 \mathrm{eV}$ and a lower bound of 0.65 for the respective thiol counterparts. These values are consistent with the respective decay coefficients described above. A summary of tunneling parameters from our analysis is shown in Table 1 . The barrier height for alkyl from our analysis is in good agreement with the theoretical values of about 3.5 to $5.0 \mathrm{eV}^{41}$ However, it is significantly higher than those reported previously for the same molecules obtained by other techniques, including PEDOT:PSS protected junctions, ${ }^{26,27}$ scanning/conductive probe techniques, ${ }^{9,20-23}$ nanopores, ${ }^{12}$ etc., all with reported values below $2.5 \mathrm{eV}$.

While results from different measurement techniques may yield different precisions and uncertainties and may need to be analyzed differently, the most critical factor in determining transport processes through MMM junctions is the nature of the interface between the molecules and the metallic contact. As reported by Wang et al., ${ }^{27}$ the current density through MMM junctions with the top Au electrodes produced by thermal evaporation directly on the molecules was determined to be 3 orders of magnitude greater than those from junctions made with an intermediate layer of PEDOT:PSS. Introduction of an intermediate conducting polymer layer is a viable approach to prevent electrical shorting from migration of $\mathrm{Au}$ through the molecules, as it was first successfully implemented by de Boer et al. ${ }^{7}$ However, in samples with a protective layer of PEDOT:PSS, the nature of the molecular barrier can be altered; for instance, the presence of PEDOT:PSS at the interface is known to lower the tunneling barrier in organic monolayers. ${ }^{64}$ In contrast, samples with direct thermal deposition of metal on top of the SAM are prone to electrical shorting, with more than 
Table 1. Summary of Tunneling Parameters Obtained from Our Multi-barrier Simmons Analysis Described in the Text ${ }^{a}$

\begin{tabular}{rlllll} 
& $\beta\left(\AA^{-1}\right)$ & $\Phi(\mathrm{eV})$ & \multicolumn{1}{c}{$\alpha$} & $d$ out-of-plane $(\mathrm{nm})$ & $\theta(\mathrm{deg})$ \\
alkyl & $1.16 \pm 0.10$ & $3.5 \pm 0.2$ & $0.6 \pm 0.1$ & $0.081 \pm 0.003$ & $41-50$ \\
thiol & $1.00 \pm 0.14$ & $2.4^{b}$ & $0.65^{c}$ & $0.30 \pm 0.02$ &
\end{tabular}

${ }^{a}$ Decay coefficient $(\beta)$, tunneling barrier height $(\Phi)$, shape parameter $(\alpha)$, and out-of-plane spacing $\left(d_{\perp}\right.$ for alkyl and $d_{\text {th }}$ for thiol) are separately listed for alkyl and thiol. The tilt angle for the alkane chain $(\theta)$ is with respect to the surface normal (Figure 5). ${ }^{b}$ Upper bound value. ${ }^{c}$ Lower bound value.

$98 \%$ of such junctions shorted in several prior reports. ${ }^{14,27}$ Furthermore, a very low value for the decay coefficient $\beta_{\text {th }}(0.05$ $\left.\AA^{-1}\right)^{52}$ indicates that the electron wave function is experiencing very little decay within the layers of thiol end groups. This and the above-mentioned enhancement in conductivity, previously reported in the literature, strongly suggest that $\mathrm{Au}$ nanostructures may have conglomerated around and covered the thiols and thus partially shorted them. It is reasonable to conclude that the interfaces in MMM junctions with the top electrodes fabricated from direct thermal deposition (including those involving nanopores) ${ }^{12,65}$ are at best ill-defined, owing to $\mathrm{Au}$ migration and conglomeration through the molecules during deposition. Similarly, contacts made with cAFM tips on molecules are also ill-defined, since the tip geometry is generally uncontrolled and unknown. In short, various interfacial defects between metal contacts and molecules produced by different fabrication processes and measurement techniques can account for the variation in the reported tunneling parameters and generally tend to lower the observed tunneling barrier.

Dependence of Tunneling Characteristics on the Area of the Printed Au Electrode. Few (if any) techniques used to fabricate molecular junctions produce scalable, permanent, and symmetrical molecular junctions. Nanotransfer printing is very unique in that it forms electrical contact directly to the self-assembled monolayer. The result is a permanent molecular junction, with very well-defined lateral dimensions (when compared with most other techniques), that is produced without the need of an additional protection layer between the printed metal electrode and the monolayer. Since the printed top electrode is presumably chemically bonded to the SAM, the environments at the two metal-molecule interfaces of the respective top and bottom $\mathrm{Au}$ electrodes are nearly identical. This is important in that it simplifies the interpretation of the observed electrical properties, allowing the transport parameters to be extrapolated for both the molecules and the metalmolecule interfaces, as we discussed above. Furthermore, nTP allows one to change the lateral dimensions of the printed top electrode (Figure 1). This feature enabled us to systematically examine the dependence of tunneling properties on the area of printed $\mathrm{Au}$ electrodes.

In practice, several hundred MMM junctions were fabricated with lateral dimensions of the top Au contacts ranging from 80 $\mathrm{nm}$ to $7 \mu \mathrm{m}$ printed on SAMs of either decanedithiol (C10) or tetradecanedithiol (C14), and the I-V behaviors of these junctions were probed by cAFM. Fifty or more separate MMM junctions were probed for each contact size from a minimum of three separately fabricated arrays. The measured tunneling current for any given bias voltage exhibits a monotonic increase with the printed contact area for the MMM junctions, as shown in Figure 7. The I-V curves scale with each other, except for the two largest pads ( $5 \mu \mathrm{m}$ squares and $7 \mu \mathrm{m}$ circles), whose high bias exponential increases are larger. The scaling of the I$\mathrm{V}$ curves is consistent with the notion that the only

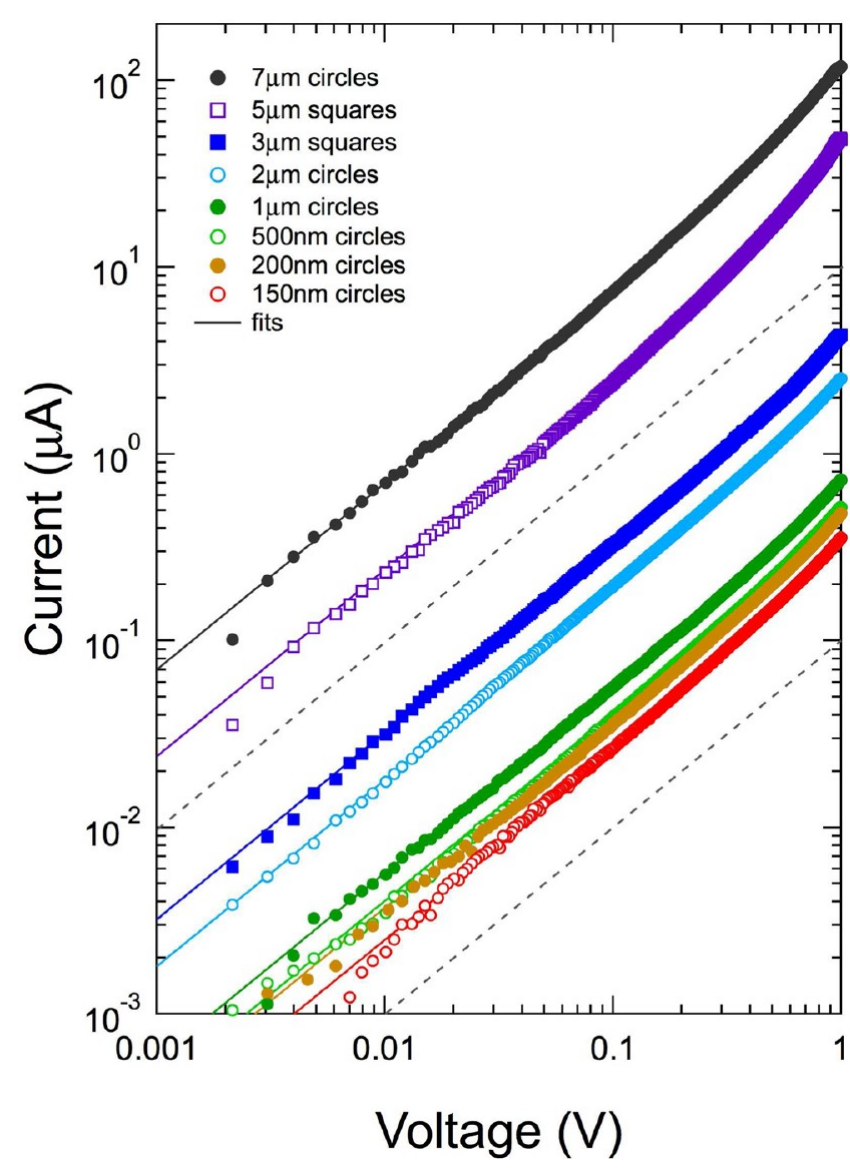

Figure 7. Dependence of tunneling current on nTP contact size printed on decanedithiol SAMs, showing a monotonic increase of the current versus contact size. Results are shown for $\mathrm{Au}$-decanedithiol$\mathrm{Au}$ junctions. The solid, colored lines corresponds to Simmons fits of the I-V curves, and the two dashed lines are guide for the eyes to indicate the linear slope at low bias.

differentiating quantity between the MMM junctions is the actual contact area. However, a close inspection of the behavior indicates that the current does not depend linearly on the area of the top printed contacts (pads), as one would expect if each pad were to contact all the molecules underneath it. Instead, the dependence of the tunneling resistance exhibits three distinct regions on the area of printed pads, two linear regions separated by a plateau, as shown in Figure 8 .

For small pads ( $\leq 200 \mathrm{~nm}$ in diameter), the low-bias resistance $R_{0}$ scales linearly with the area of the printed pads (the solid line in Figure 8 based on eq 2 using parameters obtained from our Simmons analysis). As the pad size increases above $200 \mathrm{~nm}$ in lateral dimensions, $R_{0}$ exhibits a plateau until decreasing again once the lateral dimension of the pads exceeds 1 or several $\mu \mathrm{m}$ depending on the type of PFPE stamps used. In the large pad region, $R_{0}$ appears to scale linearly with pad size again (the right dashed line in Figure 8), and the behaviors for 


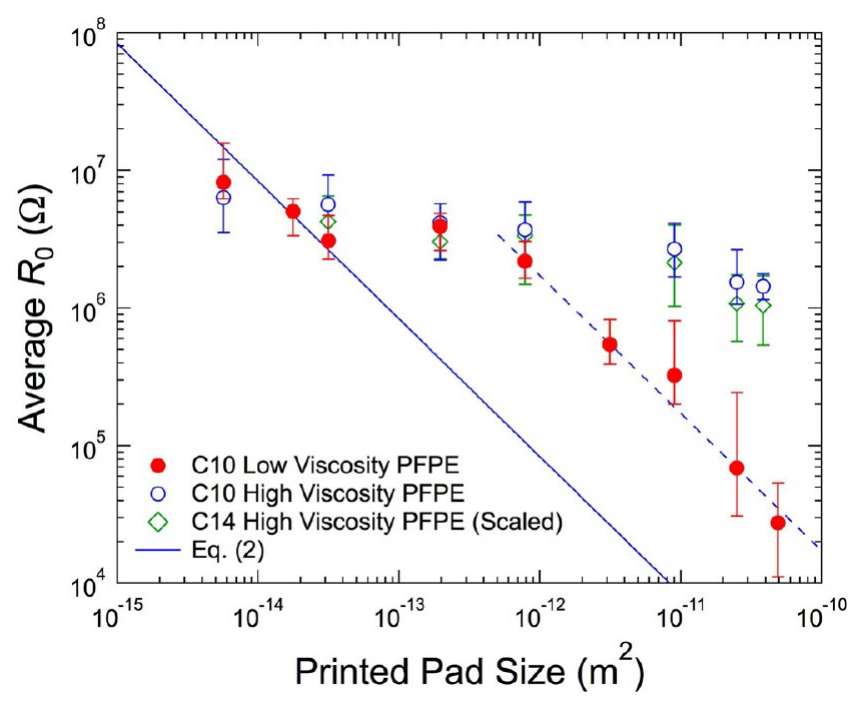

Figure 8. Dependence of zero-bias resistance $R_{0}$ on the area of the nTP pads for decanedithiol (C10) and tetradecanedithiol (C14) and for different PFPE stamps. The solid blue line is the expected dependence based on eq 2 using parameters obtained from our analysis. The dashed blue line highlights an additional linear regime for C10 junctions transfer printed using low viscosity PFPE stamps. The points for C14 are scaled down by a factor of 46 , which is equal to $\exp \left[\beta_{\mathrm{al}}(14-10) d_{\perp}\right]$ (eqs 2 and 4$)$.
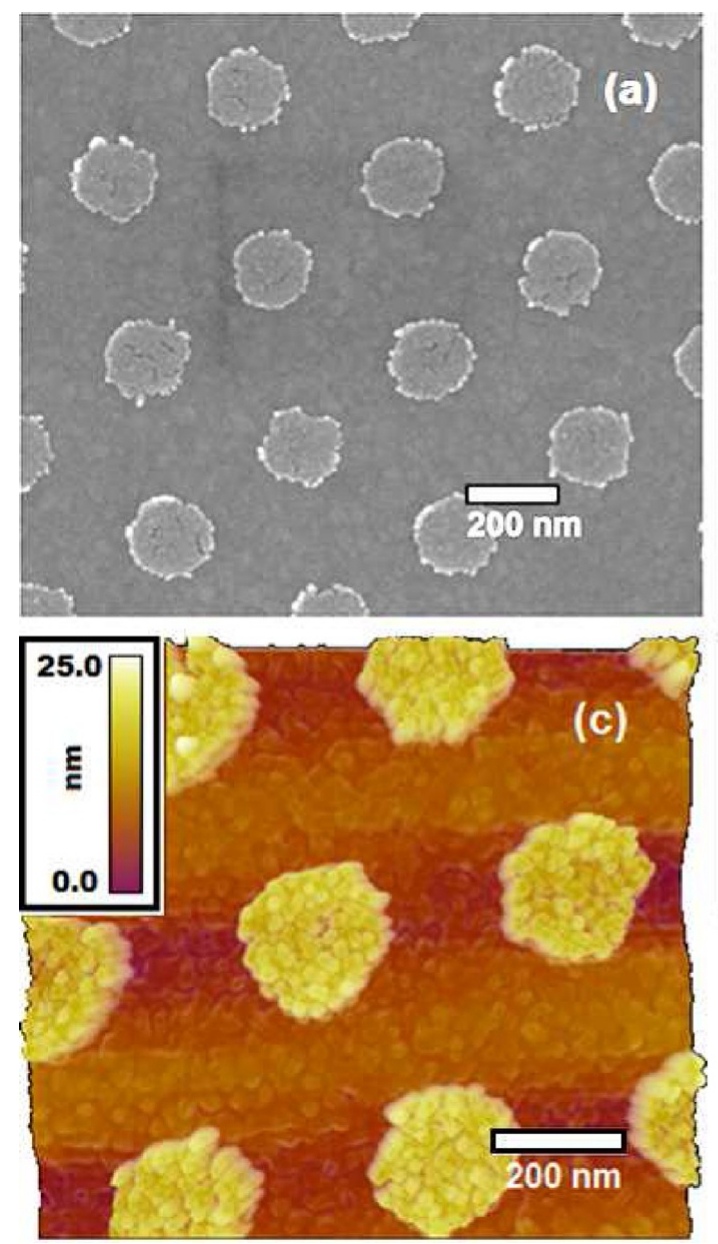

the two types of PFPE stamps used are different (closed versus open symbols in Figure 8). We note that the tunneling behaviors for $\mathrm{C} 10$ and $\mathrm{C} 14$ are both scalable with respect to printed electrode area, as discussed above (eqs 2 and 4).

There is a simple yet reasonable interpretation for the results shown in Figure 8: the effective contact area can be significantly smaller than the area of the printed pads when the pads get larger. Specifically, for small pads, the effective contact area is roughly the same as the area of the pads (the first linear region), or in other words, the pads are contacting nearly $100 \%$ of the molecules. As the pads get larger, the effective contact area first becomes constant (the plateau region) and then increases again (the second linear region), such that a fixed fraction of molecules under the pads (though $\ll 100 \%$ ) is contacted by the Au pads. This interpretation is supported by the Simmons analysis when the contact area is included as one of the fitting parameters. The notion of a small effective contact area likely means that there are two types of "contacts" within each printed pad, one bonded to the molecules and another having a "gap" between the metal pad and the molecules. To a good approximation, tunneling through the latter with a series of barriers (molecules and "gap") can be neglected, given that the measured current is likely dominated by tunneling through the molecules.

Two interesting questions arise: Which factors influence the effective contact area and its dependence on the size of printed
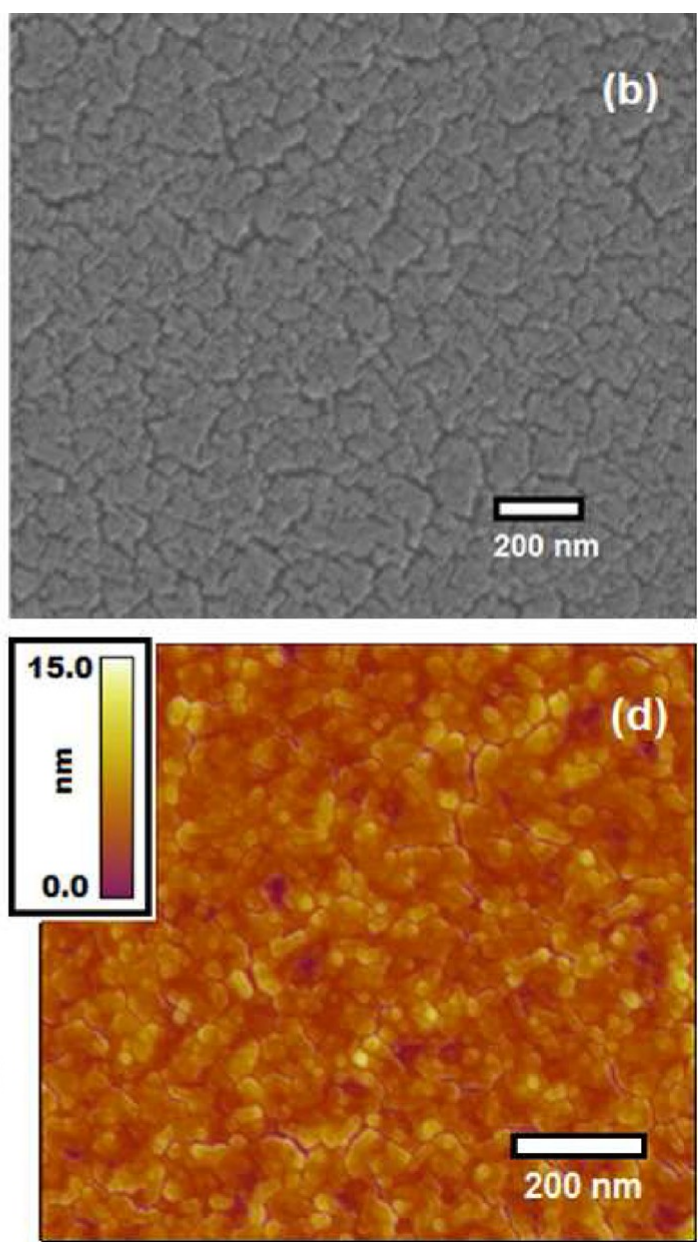

Figure 9. Characteristic surfaces of various transfer printed features. SEM images of (a) $200 \mathrm{~nm}$ diameter contacts and (b) a zoomed-in region on a 7 $\mu \mathrm{m}$ diameter printed contact. AFM topography of (c) $200 \mathrm{~nm}$ diameter features and (d) a zoomed-in region on a $5 \times 5 \mu \mathrm{m}$ printed contact. 
pads, and can these factors be controlled? We offer the following observations, starting with the contact force during the nTP process.

The primary control during the transfer process is the tapping force applied (see Experimental Section). Our experiments show that if the force is too little, no or partial transfer would occur, whereas an excessive applied force would lead to shorting between the top and bottom Au electrodes and/or overprinting in areas between the intended features. Therefore, the current optimized nTP process requires a narrow range of applied force, which also turns out to be roughly the same for all pad sizes within the limit of experimental control. As a result, the total mechanical contact force applied at the metal-molecule interface is essentially the same, since it is approximately the weight of the stamp (similar for different size pads) plus the applied tapping force. This observation suggests that within the plateau region (Figure 8), the effective contact area being constant may be the result of the contact force being constant. This argument is analogous to the physics of friction between two surfaces, whereby the frictional force is conventionally known to be proportional to the normal force and independent of the macroscopic area in contact. However, as nanotribology has revealed, the frictional force is also proportional to the microscopic contact area between the two surfaces in contact. ${ }^{66}$ Therefore, the actual contact area depends on the normal force, i.e., the same contact area is produced by the same contact force.

Like nanotribology, roughnesses of the two contacting surfaces are also important in determining the microscopic contact area. However, the nature of the interfaces as a result of the nTP process is rather complex and very challenging to elucidate experimentally. Here, our AFM and SEM experiments on surfaces before and after printing may offer some insight. As shown in Figure 9, the surfaces on small printed $\mathrm{Au}$ pads are relatively smooth with typical $R_{\text {rms }}$ values of about $4 \mathrm{~nm}$, whereas the counterparts on larger printed pads $(\geq 1 \mu \mathrm{m}$ in lateral dimensions) are considerably rougher with cracks and folded ridges. For the latter, as the number of cracks and their lengths increase with the pad size, the $R_{\mathrm{rms}}$ values also increase, from 4.4 to 5.0 to $5.5 \mathrm{~nm}$ for 3,5 , and $7 \mu \mathrm{m}$ diameter features, respectively. There is no evidence that any cracks traverse an entire pad leading to electrically isolated regions within the pad. In contrast, the $\mathrm{Au}$ surfaces prior to transfer printing, i.e., those on the bottom Au electrode and those on the PFPE stamps, are significantly smoother with typical $R_{\mathrm{rms}}$ values of $1.2-1.3 \mathrm{~nm}$, as shown in Figure S6 in the Supporting Information. The increased roughness, especially the cracking and folding, evidently arises from the relatively forceful nature of the printing process. It may also suggest that the nTP process promotes conformity at the metal-molecule interface thus deforming the top $\mathrm{Au}$ contact. There is no discernible difference in surface roughness between the Au surfaces on the two types of PFPE stamps, consistent with the comparable $R_{0}$ values (Figure 8 ) and their distributions at least for pad dimensions less than $1 \mu \mathrm{m}$ (see the histograms in Figures S4 and S5 in the Supporting Information).

Finally, we turn to the second linear region for $R_{0}$, where the behaviors for the two types of PFPE stamps deviate from each other. Again, a simple but reasonable explanation may lie in the mechanical properties of the printed Au pads and the PFPE stamps. When the pads are small, they are rigid and "plate-like", whereas large pads are analogous to flexible sheets free to conform to the surface of the SAM. The change in rigidity also explains the corresponding change in $R_{\mathrm{rms}}$ as the pad size becomes larger than $1 \mu \mathrm{m}$. Here, the increased flexibility not only lets the printed pads to be more conformal to the surface of SAM but also allows them to crack, wrinkle, and form networks of these, all of which lead to higher roughness, consistent with our microscopy measurements. The role of PFPE's elastic modulus on the nTP process is perhaps a bit subtle, and the different moduli may change how the tapping force is distributed and absorbed across the Au pads. The lowviscosity PFPE is formed from lower molecular weight precursors leading to a higher cross-link density and a higher elastic modulus $(\sim 7 \mathrm{MPa})$ when compared to those of the high-viscosity counterpart ( $\sim \mathrm{MPa})$. The stiffer stamps (lowviscosity PFPE), as opposed to the softer ones (high-viscosity PFPE), may allow the tapping force during nTP process to be more evenly distributed across each $\mathrm{Au}$ pad, resulting in a greater effective contact area and thus the lower resistance.

\section{CONCLUSIONS}

We have extensively investigated the electrical and surface properties of MMM junctions, fabricated using a novel nTP process. We show that the nTP is a reliable, nondestructive, and potentially scalable technique to produce MMM junctions with two nearly identical metal-molecule interfaces, without the need for a protection layer for the SAM. The ability to produce high quality MMM junctions has made it possible for studying electrical transport through the molecule (alkane) and the metal-molecule interfaces (thiol). The Simmons model analysis of the $\mathrm{I}-\mathrm{V}$ curves, acquired using cAFM, has yielded quantitative tunneling parameters for the molecules, including the tunneling barrier heights, $3.5 \pm 0.2 \mathrm{eV}$ (alkane) and $2.4 \mathrm{eV}$ (an upper bound value for thiol), and the corresponding decay coefficients, $1.16 \pm 0.10 \AA^{-1}$ (alkane) and $1.00 \pm 0.14 \AA^{-1}$ (thiol). These values are consistent with theoretical predictions. However, the alkane barrier height and the thiol decay coefficient are higher than those previously observed by other techniques. We attribute these differences to the improved interfacial quality between the SAM and the top printed $\mathrm{Au}$ electrode using the nTP process.

The nTP process has also made it possible to fabricate MMM junctions with a wide range of area for the top metal contacts, ranging from $80 \mathrm{~nm}$ to $7 \mu \mathrm{m}$ in lateral dimensions. Our transport measurements on junctions with different size of top contacts show that the electrical properties scale with the area of the top printed electrodes. The result also reveals the importance of the effective contact area between the printed electrode and the SAM, which can be significantly smaller than the actual area of the electrode, especially when its lateral dimension exceeds several hundred nanometers. The observed reduction in effective contact area for large contacts and its relation with the nTP parameters and surface roughness indicates the need for further optimization of the nTP process. For example, smoother interfaces (at both the PFPE stamp and the bottom electrode) would ultimately improve the printing and the resulting $\mathrm{I}-\mathrm{V}$ characteristics.

Finally, we have demonstrated that the MMM junctions and arrays, fabricated by the soft lithography assisted nTP, provide a useful and reliable test bed for investigating outstanding issues in the science and technology of molecular electronics. 


\section{ASSOCIATED CONTENT}

\section{S Supporting Information}

Device fabrication and experimental details. This information is available free of charge via the Internet at http://pubs.acs.org.

\section{AUTHOR INFORMATION}

\section{Corresponding Author}

wyou@unc.edu

\section{Notes}

The authors declare no competing financial interest.

\section{ACKNOWLEDGMENTS}

This work was supported by a NSF CCI Award (CHE0943975). W.C.R. acknowledges fellowship support from NSF GAANN. We acknowledge Professor J. M. DeSimone and the DeSimone group for supplying PFPE molds.

\section{REFERENCES}

(1) Heath, J. R. Annu. Rev. Mater. Res. 2009, 39, 1-23.

(2) Akkerman, H. B.; de Boer, B. J. Phys.: Condensed Matter 2008, 20, 013001.

(3) Mann, B.; Kuhn, H. J. Appl. Phys. 1971, 42, 4398-4405.

(4) Chen, F.; Hihath, J.; Huang, Z.; Li, X.; Tao, N. J. Annu. Rev. Phys. Chem. 2007, 58, 535-564.

(5) Kelley, T. W.; Granstrom, E.; Frisbie, C. D. Adv. Mater. 1999, 11, 261-264.

(6) Loo, Y.-L.; Lang, D. V.; Rogers, J. A.; Hsu, J. W. P. Nano Lett. 2003, 3, 913-917.

(7) Akkerman, H. B.; Blom, P. W. M.; de Leeuw, D. M.; de Boer, B. Nature 2006, 441, 69-72.

(8) Chiechi, R. C.; Weiss, E. A.; Dickey, M. D.; Whitesides, G. M. Angew. Chem., Int. Ed. 2008, 47, 142-144.

(9) Li, C.; Pobelov, I.; Wandlowski, T.; Bagrets, A.; Arnold, A.; Evers, F. J. Am. Chem. Soc. 2008, 130, 318-326.

(10) de Boer, B.; Frank, M. M.; Chabal, Y. J.; Jiang, W.; Garfunkel, E.; Bao, Z. Langmuir 2004, 20, 1539-1542.

(11) Chen, J.; Reed, M. A.; Rawlett, A. M.; Tour, J. M. Science 1999, 286, 1550-1552.

(12) Wang, W.; Lee, T.; Reed, M. A. Phys. Rev. B 2003, 68, 035416.

(13) Wang, W.; Lee, T.; Kretzschmar, I.; Reed, M. A. Nano Lett. 2004, 4, 643-646.

(14) Kim, T.-W.; Wang, G.; Lee, H.; Lee, T. Nanotechnology 2007, 18,315204 .

(15) Reed, M. A.; Zhou, C.; Muller, C. J.; Burgin, T. P.; Tour, J. M. Science 1997, 278, 252-254.

(16) Rampi, M. A.; Schueller, O. J. A.; Whitesides, G. M. Appl. Phys. Lett. 1998, 72, 1781-1783.

(17) York, R. L.; Nguyen, P. T.; Slowinski, K. J. Am. Chem. Soc. 2003, 125, 5948-5953.

(18) Weiss, E. A.; Chiechi, R. C.; Kaufman, G. K.; Kriebel, J. K.; Li, Z.; Duati, M.; Rampi, M. A.; Whitesides, G. M. J. Am. Chem. Soc. 2007, $129,4336-4349$.

(19) Kushmerick, J. G.; Holt, D. B.; Yang, J. C.; Naciri, J.; Moore, M. H.; Shashidhar, R. Phys. Rev. Lett. 2002, 89, 086802.

(20) Li, X.; He, J.; Hihath, J.; Xu, B.; Lindsay, S. M.; Tao, N. J. Am. Chem. Soc. 2006, 128, 2135-2141.

(21) Wold, D. J.; Haag, R.; Rampi, M. A.; Frisbie, C. D. J. Phys. Chem. B 2002, 106, 2813-2816.

(22) Wang, G.; Kim, T.-W.; Jo, G.; Lee, T. J. Am. Chem. Soc. 2009, $131,5980-5985$.

(23) Engelkes, V. B.; Beebe, J. M.; Frisbie, C. D. J. Am. Chem. Soc. 2004, 126, 14287-14296.

(24) Salomon, A.; Cahen, D.; Lindsay, S.; Tomfohr, J.; Engelkes, V. B.; Frisbie, C. D. Adv. Mater. 2003, 15, 1881-1890.
(25) Akkerman, H. B.; Kronemeijer, A. J.; Harkema, J.; van Hal, P. A.; Smits, E. C. P.; de Leeuw, D. M.; Blom, P. W. M. Org. Electron. 2008, $11,146-149$.

(26) Akkerman, H. B.; Naber, R. C. G.; Jongbloed, B.; van Hal, P. A.; Blom, P. W. M.; de Leeuw, D. M.; de Boer, B. Proc. Natl. Acad. Sci. U.S.A. 2007, 104, 11161-11166.

(27) Wang, G.; Yoo, H.; Na, S.-I.; Kim, T.-W.; Cho, B.; Kim, D.-Y.; Lee, T. Thin Solid Films 2009, 518, 824-828.

(28) Wang, G.; Kim, Y.; Choe, M.; Kim, T.-W.; Lee, T. Adv. Mater. 2011, 23, 755-760.

(29) Loo, Y.-L.; Willett, R. L.; Baldwin, K. W.; Rogers, J. A. Appl. Phys. Lett. 2002, 81, 562-564.

(30) Loo, Y.-L.; Willett, R. L.; Baldwin, K. W.; Rogers, J. A. J. Am. Chem. Soc. 2002, 124, 7654-7655.

(31) Lee, B. H.; Cho, Y. H.; Lee, H.; Lee, K. D.; Kim, S. H.; Sung, M. M. Adv. Mater. 2007, 19, 1714-1718.

(32) Kaoru, O.; Yoichi, O.; Takuya, M.; Tomoji, K.; Kenichiro, N.; Shinji, M. Appl. Phys. Lett. 2005, 87, 234110.

(33) Guerin, D.; Merckling, C.; Lenfant, S.; Wallart, X.; Pleutin, S.; Vuillaume, D. J. Phys. Chem. C 2007, 111, 7947-7956.

(34) Kim, J.-W.; Yang, K.-Y.; Hong, S.-H.; Lee, H. Appl. Surf. Sci. 2008, 254, 5607-5611.

(35) Loo, Y.-L.; Hsu, J. W. P.; Willett, R. L.; Baldwin, K. W.; West, K. W.; Rogers, J. A. J. Vac. Sci. Technol. B 2002, 20, 2853-2856.

(36) Wang, R. Y.; Segalman, R. A.; Majumdar, A. Appl. Phys. Lett. 2006, 89, 173113.

(37) Felmet, K.; Loo, Y.-L.; Sun, Y. Appl. Phys. Lett. 2004, 85, 33163318.

(38) Jiang, W.; Garfunkel, E.; Zhitenev, N.; Abusch-Magder, D.; Tennant, D.; Bao, Z. Appl. Phys. Lett. 2006, 89, 113107.

(39) Fan, X.; Rogow, D. L.; Swanson, C. H.; Tripathi, A.; Oliver, S. R. J. Appl. Phys. Lett. 2007, 90, 163114.

(40) Niskala, J. R.; You, W. J. Am. Chem. Soc. 2009, 131, 1320213203.

(41) Tomfohr, J. K.; Sankey, O. F. Phys. Rev. B 2002, 65, 245105.

(42) Vericat, C.; Vela, M. E.; Benitez, G.; Carro, P.; Salvarezza, R. C. Chem. Soc. Rev. 2010, 39, 1805-1834.

(43) Love, J. C.; Estroff, L. A.; Kriebel, J. K.; Nuzzo, R. G.; Whitesides, G. M. Chem. Rev. 2005, 105, 1103-1170.

(44) Hoertz, P. G.; Niskala, J. R.; Dai, P.; Black, H. T.; You, W. J. Am. Chem. Soc. 2008, 130, 9763-9772.

(45) Millone, M. a. A. D.; Hamoudi, H.; Rodríguez, L.; Rubert, A.; Benítez, G. A.; Vela, M. a. E.; Salvarezza, R. C.; Gayone, J. E.; Sánchez, E. A.; Grizzi, O.; Dablemont, C. 1.; Esaulov, V. A. Langmuir 2009, 25, 12945-12953.

(46) Akkerman, H.e B.; Kronemeijer, A. J.; van Hal, P. A.; de Leeuw, D. M.; Blom, P. W. M.; de Boer, B. Small 2008, 4, 100-104.

(47) Rieley, H.; Kendall, G. K.; Zemicael, F. W.; Smith, T. L.; Yang, S. Langmuir 1998, 14, 5147-5153.

(48) Carot, M. L.; Esplandiu, M. J.; Cometto, F. P.; Patrito, E. M.; Macagno, V. A. J. Electroanal. Chem. 2005, 579, 13-23.

(49) Rolland, J. P.; Hagberg, E. C.; Denison, G. M.; Carter, K. R.; Simone, J. M. D. Angew. Chem., Int. Ed. 2004, 43, 5796-5799.

(50) Simmons, J. G. J. Appl. Phys. 1963, 34, 1793-1803.

(51) Simmons, J. G. J. Appl. Phys. 1963, 34, 2581-2590.

(52) Wang, G.; Kim, T.-W.; Jang, Y. H.; Lee, T. J. Phys. Chem. C 2008, 112, 13010-13016.

(53) Poirier, G. E. Chem. Rev. 1997, 97, 1117-1128.

(54) Schreiber, F. Prog. Surf. Sci. 2000, 65, 151-257.

(55) Ulman, A. Chem. Rev. 1996, 96, 1533-1554.

(56) Luca, M. G.; Riccarda, C.; Luigi Delle, S. J. Phys.: Condens. Matter 2007, 19, 176004.

(57) Barrena, E.; Ocal, C.; Salmeron, M. J. Chem. Phys. 2000, 113, 2413-2418.

(58) Barrena, E.; Ocal, C.; Salmeron, M. J. Chem. Phys. 2001, 114, $4210-4214$

(59) Slowinski, K.; Chamberlain, R. V.; Miller, C. J.; Majda, M. J. Am. Chem. Soc. 1997, 119, 11910-11919. 
(60) Wang, G.; Kim, T.-W.; Lee, H.; Lee, T. Phys. Rev. B 2007, 76, 205320.

(61) Xu, B.; Tao, N. J. Science 2003, 301, 1221-1223.

(62) Wold, D. J.; Frisbie, C. D. J. Am. Chem. Soc. 2001, 123, 55495556.

(63) Cui, X. D.; Zarate, X.; Tomfohr, J.; Sankey, O. F.; Primak, A.; Moore, A. L.; Moore, T. A.; Gust, D.; Harris, G.; Lindsay, S. M. Nanotechnology 2002, 13, 5-14.

(64) Koch, N.; Kahn, A.; Ghijsen, J.; Pireaux, J. J.; Schwartz, J.; Johnson, R. L.; Elschner, A. Appl. Phys. Lett. 2003, 82, 70-72.

(65) Song, H.; Lee, T.; Choi, N.-J.; Lee, H. Appl. Phys. Lett. 2007, 91, 253116.

(66) Krim, J. Sci. Am. 1996, 275, 74-80. 